\title{
QT-RR Relation Is Different in Humans and Rats
}

\author{
Beatrice De Maria ${ }^{1}$, Vlasta Bari ${ }^{2}$, Beatrice Cairo ${ }^{3}$, Aparecida Maria Catai ${ }^{4}$, Anielle Cristhine de \\ Medeiros Takahashi ${ }^{4}$, Luca Carnevali ${ }^{5,6}$, Andrea Sgoifo ${ }^{5,6}$, Francesca Perego ${ }^{1}$, Laura Adelaide Dalla \\ Vecchia $^{1}$, Alberto Porta ${ }^{2,3}$ \\ ${ }^{1}$ IRCCS Istituti Clinici Scientifici Maugeri, Milan, Italy \\ ${ }^{2}$ Department of Cardiothoracic, Vascular Anesthesia and Intensive Care, IRCCS Policlinico San \\ Donato, Milan, Italy \\ ${ }^{3}$ Department of Biomedical Sciences for Health, University of Milan, Milan, Italy \\ ${ }^{4}$ Department of Physiotherapy, Federal University of São Carlos, São Carlos, Brazil \\ ${ }^{5}$ Stress Physiology Laboratory, Department of Chemistry, Life Sciences and Environmental \\ Sustainability, University of Parma, Parma, Italy \\ ${ }^{6}$ Microbiome Research Hub, University of Parma, Parma, Italy
}

\begin{abstract}
The QT interval (QT) variability has been recently computed to infer cardiac control of rats. It has been suggested that $Q T$ variability markers in rats have the same physiological meaning as in humans. However, some evidences indicate a different dependence of $Q T$ on the previous RR interval (RR).

Thus, the aim of this study was to compare the relation of the $Q T$ to the preceding $R R$ in humans and in rats.

Electrocardiogram was recorded in supine position (REST) and during tilt test (T90) in 23 healthy subjects and in 9 Wistar (WI) and 14 wild-type Groningen (WT) rats during the dark period. Pearson product moment correlation coefficient $r$ computed between RR and QT was calculated for each subject or animal within each experimental condition.

In humans we found that $r$ was positive and decreased from REST to T90. Conversely, $r$ was negative in rats and did not differ between WI and WT. The $r$ absolute value was significantly higher in humans than in rats. Our results showed that trends toward longer RRs lead to longer QTs in humans but shorter QTs in rats and that the strength of the QT-RR association is lower in rats.

We conclude that attention should be paid when using the rat model in translational studies assessing the $Q T-R R$ relation.
\end{abstract}

\section{Introduction}

In the last years QT interval (QT) variability markers have been added to those computed over the spontaneous fluctuations of RR interval (RR) [1-6]. The rationale underlying this strategy is that the QT variability analysis seems to provide additional information about the cardiac neural control since QT variability is more useful to assess neural control directed to ventricles, while RR variability is more suitable for the evaluation of neural regulation directed to the sinus node [3]. In humans, part of the QT variability is dependent on $R R$ variations given the strong link of QT with the preceding RR [5,7].

Only recently the QT variability has been successfully exploited in rats for the assessment of the cardiac control. It was suggested that QT variability has the same physiological meaning in humans as in rats [1]. This observation seems to hold despite the difference between humans and rats concerning the response of the QT to the RR shortening induced by a sympathetic stimulus: indeed, rats respond to sympathetic stimulation with a prolongation of the QT interval, while humans with a QT shortening [8,9].

Thus, the aim of this study is to evaluate the relation of QT to the preceding RR in humans and in rats. The hypothesis is that, despite the presence of similar physiological interpretation of both RR and QT variabilities, the relation between RR and QT is different in humans and rats. In humans, the relation between RR and QT is studied in young healthy subjects performing a maneuver inducing a sympathetic activation and vagal withdrawal, namely the head-up tilt test. In rats, the relation between QT and RR is studied in two different rat strains known to exhibit different social traits that are mirrored by diverse autonomic states at rest, namely the Wistar (WI) rats and wild-type Groningen (WT) rats [10].

\section{Experimental protocols}


TABLE 1. TIME DOMAIN RR AND QT VARIABILITY INDEXES OF THE HUMAN PROTOCOL

\begin{tabular}{lcc}
\hline \multicolumn{1}{c}{ Index } & REST & T90 \\
\hline$\mu_{\mathrm{RR}}[\mathrm{ms}]$ & $937.18 \pm 135.11$ & $715.18 \pm 96.47^{*}$ \\
$\mu_{\mathrm{QT}}[\mathrm{ms}]$ & $327.91 \pm 33.6$ & $295.09 \pm 30.19^{*}$ \\
$\sigma_{\mathrm{RR}}\left[\mathrm{ms}^{2}\right]$ & $2755.2 \pm 2083.51$ & $1773.36 \pm 940.24$ \\
$\sigma^{2}{ }_{\mathrm{QT}}\left[\mathrm{ms}^{2}\right]$ & $11.43 \pm 12.29$ & $23.21 \pm 33.49$ \\
\hline
\end{tabular}

REST: at rest in supine position; T90: head-up tilt at $90^{\circ}$; RR: RR interval; QT: QT interval; $\mu_{\mathrm{RR}}$ : RR mean; $\mu_{\mathrm{QT}}$ : QT mean; $\sigma^{2}{ }_{\mathrm{RR}}$ : RR variance; $\sigma^{2} \mathrm{QT}$ : QT variance. The symbol * indicates $p<0.05$ versus REST.

Two different experimental protocols were utilized in this study, one of young healthy human subjects and one of rats. A full description of the protocols was given elsewhere [1]. Briefly, in the human protocol we enrolled 23 young healthy subjects ( 11 males, age $26.3 \pm 5.6$ years). Subjects were asked to avoid alcoholic and caffeinated beverages in the 24 hours preceding the test. Electrocardiogram (ECG) from modified lead II was acquired with a sampling rate of $1000 \mathrm{~Hz}$ (Biosignal Conditioning Device, Marazza, Monza, Italy) for 10 minutes at rest in supine position (REST) and for 10 minutes during head-up tilt test with tilt table inclination at $90^{\circ}$ (T90). Attention was paid in positioning the electrodes to obtain an adequate T-wave. All the subjects completed the test without any sign of presyncope. The study adhered to the principles of the Declaration of Helsinki and was approved by the L. Sacco Hospital ethics committee. All the subjects signed a written informed consent before the starting of the experimental protocol.

In the animal protocol, we monitored 9 male WI rats (age: $5.5 \pm 0.5$ months; weight: $436 \pm 34 \mathrm{~g}$ ) and 14 male WT rats (age: $4.4 \pm 0.5$ months; weight: $395 \pm 40 \mathrm{~g}$ ). Rats were individually housed with controlled temperature and light (lights on from 7:00 P.M. to 7 A.M.) and implanted with a radio-telemetric transmitter (TA11CTA-F40, Data Sciences International, St. Paul, MN, United States) while anesthetized. After 14 days from surgery, ECG was recorded for 1 hour during the dark period by the platform receiver (RPC-1 and ART-Gold 4.2 data acquisition system, Data Sciences International, St. Paul, MN, United States) placed under the animal's cage. ECG was sampled at $1000 \mathrm{~Hz}$. The animal experimental protocol was approved by the Veterinarian Animal Care and Use Committee of the University of Parma, Parma, Italy, and the animals were cared in accordance with the European Community Council Directives (2010/63/UE).

\section{Methods}

\subsection{RR and QT beat-to-beat series extraction}

In both human and animal protocols, the RR and QT
TABLE 2. TIME DOMAIN RR AND QT VARIABILITY INDEXES OF THE ANIMAL PROTOCOL

\begin{tabular}{lcc}
\hline \multicolumn{1}{c}{ Index } & WI & WT \\
\hline$\mu_{\mathrm{RR}}[\mathrm{ms}]$ & $169.99 \pm 7.37$ & $185.6 \pm 8.31$ \\
$\mu_{\mathrm{QT}}[\mathrm{ms}]$ & $59.54 \pm 2.21$ & $56.23 \pm 2.32 \#$ \\
$\sigma_{\mathrm{RR}}\left[\mathrm{ms}^{2}\right]$ & $23.76 \pm 19.62$ & $21.38 \pm 15.5$ \\
$\sigma^{2}{ }_{\mathrm{QT}}\left[\mathrm{ms}^{2}\right]$ & $2.98 \pm 3.49$ & $2.95 \pm 6.01$ \\
\hline
\end{tabular}

WI: Wistar rats; WT: Wild-type Groningen rats; RR: RR interval; QT: QT interval; $\mu_{\mathrm{RR}}$ : RR mean; $\mu_{\mathrm{QT}}$ : QT mean; $\sigma^{2}{ }_{R R}$ : RR variance; $\sigma^{2} \mathrm{QT}: \mathrm{QT}$ variance. The symbol \# indicates $p<0.05$ versus WI.

beat-to-beat series were obtained from the recorded ECG. The time distance between two consecutive R-wave peaks was considered as the RR interval. The R-wave apexes were fixed by parabolic interpolation. The QT interval was approximated as the temporal distance between the Rwave peak and the end of the T-wave [11]. The offset of the T-wave was set where the absolute value of the first derivative of the descending part of the T-wave became lower than the $30 \%$ of its maximum value calculated over the T-wave downslope [11]. The $i$ th QT [i.e. QT $(i)$ ] started from the second R-wave delimiting the offset of the $i$ th RR [i.e. $\mathrm{RR}(i)$ ], where $i$ is the progressive measure counter.

The detections of the fiduciary points (i.e. R-wave peak and $\mathrm{T}$-wave end) were visually checked to avoid errors. Ectopic beats or artifacts were corrected by means of linear interpolation between measurements unaffected by ectopics or artifacts. Attention was paid to never correct more than $5 \%$ of the total considered measures.

As to the human protocol, stationary segments of 300 consecutive beats were selected for further analysis for each experimental condition (i.e. REST and T90). As to the animal protocol, stationary segments of 2000 beats were selected. The segments were as much as possible long according to [12] within each experimental condition. The lengths of the series in human and animal protocols were similar when expressed in absolute time given that mean RR was about five times shorter in rat than in man. In rats we carried out also an additional analysis that considered sequences of 300 beats extracted at random from the overall segment. Over the selected RR and QT series we calculated mean and variance. We indicated them as $\mu_{\mathrm{RR}}$, $\mu_{\mathrm{QT}}, \sigma_{\mathrm{RR}}^{2}$ and $\sigma_{\mathrm{QT}}^{2}$. Means were expressed in ms, while variances in $\mathrm{ms}^{2}$.

\subsection{Evaluation of QT-RR relation}

We estimated the linear relation of $\mathrm{QT}(i)$ to $\mathrm{RR}(i)$ according to [12]. The strength of this relation was assessed via the Pearson product moment correlation coefficient $r$. We checked the significance of the association between $\mathrm{RR}(i)$ and $\mathrm{QT}(i)$ by computing the probability $p$ of type I error associated with $r$. A $p<0.05$ was considered to be significant. The same analysis was 


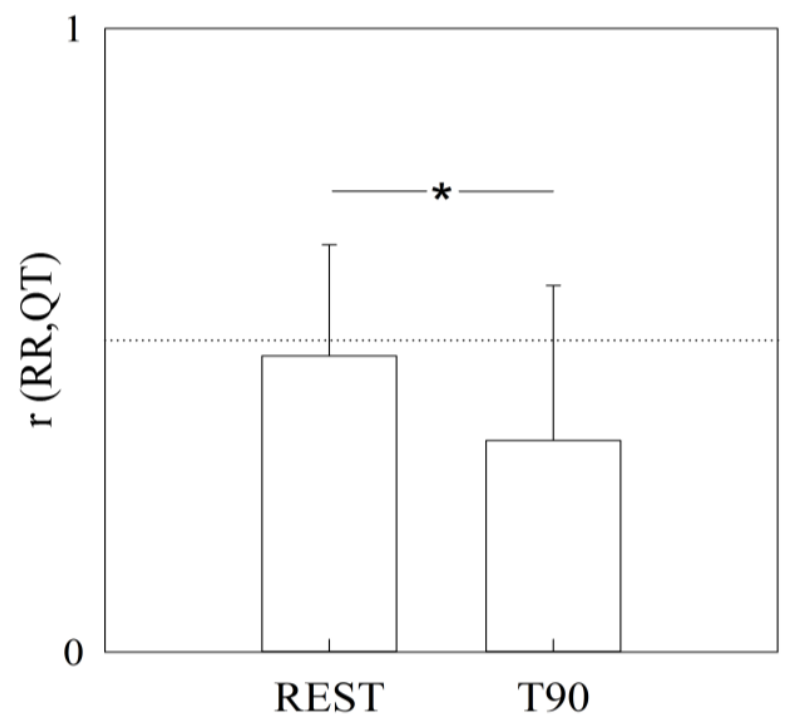

Figure 1. Comparison of $r$ computed in the plane $[\operatorname{RR}(i), \mathrm{QT}(i)]$ in healthy subjects at REST and during T90. The results are presented as mean \pm standard deviation. The dotted line denotes $r=0.5$. The symbol $*$ indicates $p<0.05$ REST vs T90.

repeated by computing the Spearman rank correlation coefficient $\rho$ to check for eventual discrepancies due to non-normal distributions of RR and QT series.

\subsection{Statistical analysis}

The effect of T90 in humans was tested by paired t test, or Wilcoxon signed rank test, when appropriate. The difference between strains in the animal protocol was tested by unpaired t test, or Mann-Whitney rank sum test, when appropriate. Results were always presented as mean \pm standard deviation. Statistical analysis was carried out using a commercial statistical program (Sigmaplot, Systat Software, Inc., Chicago, IL, United States, version 11.0). A $p<0.05$ was considered as significant.

\section{Results}

The results of the time domain analysis in humans are shown in Tab.1. $\mu_{\mathrm{RR}}$ and $\mu_{\mathrm{QT}}$ both decreased during T90. No statistically significant variation was observed in $\sigma^{2}{ }_{R R}$ and $\sigma^{2}$ QT. The results of time domain analysis in rats are shown in Tab.2. $\mu_{\mathrm{RR}}$ was similar in WI and WT rats, but $\mu_{\mathrm{QT}}$ was lower in WT rats compared to WI. WI and WT exhibited alike $\sigma_{\mathrm{RR}}^{2}$ and $\sigma_{\mathrm{QT}}^{2}$.

Pearson correlation coefficient $r$ computed in the young healthy subjects is shown in Fig. 1 as a function of the experimental condition (i.e. REST and T90). $r$ was positive in both the experimental conditions and significantly decreased during T90 compared to REST. The degree of QT-RR association was significant in $96 \%$ of the subjects at REST and in $92 \%$ during T90.

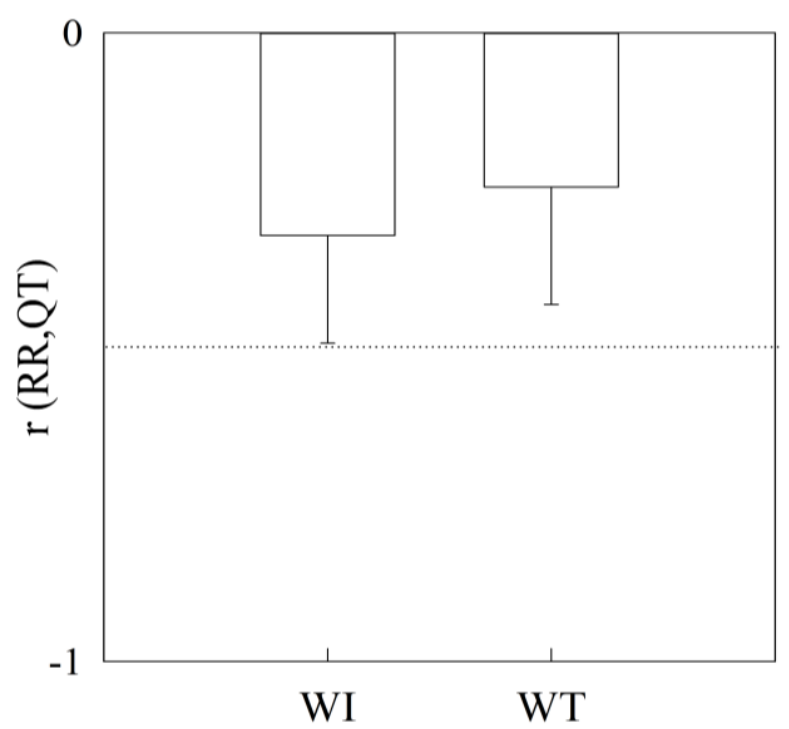

Figure 2. Comparison of $r$ computed in the plane $[\mathrm{RR}(i), \mathrm{QT}(i)]$ computed over WI and WT rats. The results are presented as mean \pm standard deviation. The dotted line denotes $r=-0.5$.

Pearson correlation coefficient $r$ computed in the rat strains is shown in Fig.2. It was assessed over the overall RR and QT series. $r$ was negative in both the strains and did not differ between WI and WT. The QT-RR correlation was statistically significant in $100 \%$ of the WI rats and in $93 \%$ of the WT rats. Similar results were obtained when we considered sequences of 300 consecutive measures but in this case QT and RR were not significantly associated in about $30 \%$ of animals.

The absolute value of $r$ was significantly higher in humans than in rats $(0.41 \pm 0.22$ vs $0.27 \pm 0.18)$, when data were pooled together regardless of the experimental condition or rat strain. This conclusion held even when $r$ was computed over sequences of 300 consecutive measures in the rat protocol $(0.41 \pm 0.22$ vs $0.19 \pm 0.16)$.

The results of the QT-RR analysis carried out via Spearman correlation coefficient $\rho$ was not shown given that results were similar to those derived from Pearson correlation coefficient $r$.

\section{Discussion}

The main novelty of this study lies in the evaluation of the QT-RR relation in rats by means of an automatic assessment of QT and RR variability and in the comparison of the QT-RR correlation in humans and rats. The main findings of this study can be summarized as follow: i) the correlation coefficient of the QT-RR relation is positive in humans, but negative in rats; ii) the degree of correlation is weaker in rats than in humans; iii) the degree of the QTRR association decreased during the sympathetic activation induced by head up tilt test; iv) the degree of the QT-RR association did not differ between WI and WT rats. 
Our study first demonstrates using beat-to-beat variability series extracted automatically from ECG recordings that the QT-RR relation differs profoundly between humans and rats. Indeed, the sign of the correlation between QT and the previous RR is opposite in the two species. The strength of the QT-RR correlation is influenced by the sympathetic challenge, as demonstrated by its decrease during head-up tilt test in humans. A decrease of the QT-RR strength during orthostatic challenge was reported in the frequency and information domain as well $[5,13]$. The lack of difference between WI and WT could be attributable to the known sympathetic dominance in rats [14], that could also explain the weaker correlation between RR and QT in rats compared to humans. Our results are corroborated by studies carried out on manual RR and QT measurements in rats [15-17] showing that in rats the sign of the QT-RR correlation could be affected by drugs [15] and that the QT has negligible variations in presence of important RR changes induced by pharmacological challenges [17].

The main limitation of the present study lies in the lack of a sympathetic challenge in the animal protocol. In addition, the choice of a simple linear model for the evaluation of the QT-RR relation could be considered an additional restraint. Indeed, the QT-RR relation has been demonstrated to exhibit nonlinear terms [18]. Future studies are needed to confirm the results of the present study with more adequate dynamical models of the QT-RR relation accounting for the dynamical dependences of QT on several RRs $[3,13]$.

\section{Conclusions}

In this study we evaluated the QT-RR correlation in healthy young subjects performing head-up tilt test and in different rat strains. Results showed that the correlation coefficient of the relation of QT to the preceding RR is of opposite sign in humans and rats. We conclude that attention should be paid in the use of the rat model in translational studies assessing the QT-RR relation.

\section{References}

[1] B. De Maria et al., "Concomitant evaluation of heart period and QT interval variability spectral markers to typify cardiac control in humans and rats," Front. Physiol., vol. 10, 2019, Art. no. 1478.

[2] M. Baumert et al., "QT interval variability in body surface ECG: measurement, physiological basis, and clinical value: position statement and consensus guidance endorsed by the European Heart Rhythm Association jointly with the ESC Working Group on Cardiac Cellular Electrophysiology," Europace, vol. 18, pp. 925-944, 2016.

[3] A. Porta, E. Tobaldini, T. Gnecchi-Ruscone and N. Montano, "RT variability unrelated to heart period and respiration progressively increases during graded head-up tilt," Am. J. Physiol., vol. 298, pp. H1406-H1414, 2010.
[4] M. Baumert et al., "Relation between QT interval variability and cardiac sympathetic activity in hypertension," Am. J. Physiol., vol. 300, pp. H1412-H1427, 2011.

[5] A. Porta et al., "Frequency domain assessment of the coupling strength between ventricular repolarization duration and heart period during graded head-up tilt," $J$. Electrocardiol., vol. 44, pp. 662-668, 2011.

[6] R. D. Berger, "QT interval variability is it a measure of autonomic activity?" J. Am. Coll. Cardiol., vol. 54, pp. 851852, 2009.

[7] H. C. Bazett, "An analysis of the time-relations of electrocardiograms," Heart, vol. 7, pp. 353-370, 1929.

[8] T. Speerschneider and M. B. Thomsen, "Physiology and analysis of the electrocardiographic T wave in mice," Acta Physiol., vol. 209, pp. 262-271, 2013.

[9] C. E. Conrath and T. Opthof, "Ventricular repolarization: an overview of (patho)physiology, sympathetic effects and genetic aspects," Prog. Biophys. Mol. Biol., vol. 92, pp. 269307, 2006.

[10] L. Carnevali and A. Sgoifo, "Vagal modulation of resting heart rate in rats: the role of stress, psychosocial factors, and physical exercise," Front. Physiol., vol. 5, pp. 118, 2014.

[11] A. Porta et al., "Performance assessment of standard algorithms for dynamic R-T interval measurement: comparison between R-Tapex and R-Tend approach," Med. Biol. Eng. Comput., vol. 36, pp. 35-42, 1998.

[12] M. Merri et al., "Relation between ventricular repolarization duration and cardiac cycle length during 24-hour Holter recordings. Findings in normal patients and patients with long QT syndrome," Circulation, vol. 85, pp. 1816-1821, 1992.

[13] A. Porta, V. Bari, B. De Maria and M. Baumert, "A network physiology approach to the assessment of the link between sinoatrial and ventricular cardiac controls," Physiol. Meas., vol. 38, pp. 1472-1489, 2017.

[14] T. Opthof, "The normal range and determinants of the intrinsic heart rate in man," Cardiovasc. Res., vol. 45, pp. 177-184, 2000.

[15] S. A. Ben-Haim, H. Ben-Ami, G. Hayam, U. Taitelman and Y. Edoute, "Effect of phosphamidon and obidoxime on the QT-RR relationship of isolated rat heart," Pharmacol. Toxicol., vol. 70, pp. 402-406, 1992.

[16] E. Hayes, M. K. Pugsley, W. P. Penz, G. Adaikan and M. J. Walker, "Relationship between QaT and RR intervals in rats, guinea pigs, rabbits, and primates," J. Pharmacol. Toxicol. Methods, vol. 32, pp. 201-207, 1994.

[17] J. Kmecova and J. Klimas, "Heart rate correction of the QT duration in rats," Eur. J. Pharmacol., vol. 641, pp. 187-192, 2010.

[18] E. Pueyo et al., "Characterization of QT interval adaptation to RR interval changes and its use as a risk-stratifier of arrhythmic mortality in amiodarone-treated survivors of acute myocardial infarction," IEEE Trans. Biomed. Eng., vol. 51, pp. 1511-1520, 2004.

Address for correspondence:

Beatrice De Maria, PhD

IRCCS Istituti Clinici Scientifici Maugeri

Via Camaldoli 64, 20138, Milan, Italy.

Phone: +390250725286

Email: beatrice.demaria@icsmaugeri.it 